age at leaving work and suicide/overdose were null. Yet, among miners with post-1980 job loss, leaving work before age 30 and between ages $30-40$ increased the risk of suicide/ overdose compared with leaving work after age 55 (1.94 $(1.05,3.58)$ and $1.46(0.78,2.72)$, respectively).

Conclusions Our results suggest an elevated risk of suicide among male miners in the $1980 \mathrm{~s}$, and that the risk increases after leaving work.

\section{S-243 DEATH BY ROBOTS? AUTOMATION AND WORKING-AGE MORTALITY IN THE UNITED STATES}

${ }^{1}$ Atheendar Venkataramani, Rourke O'Brien, Elizabeth Bair. 'University of Pennsylvania, United States

\subsection{6/OEM-2021-EPI.426}

The decline of manufacturing employment is frequently invoked as a key cause of worsening U.S. population health trends, including increased mortality due to rising 'deaths of despair'. Increasing automation - the use of industrial robots to perform tasks previously done by human workers-is one major structural force driving the decline of manufacturing jobs and wages. In this study we examine the impact of automation on age-sex specific mortality. Using exogenous variation in automation to support causal inference, we find that increases in automation over the period 1993-2007 led to substantive increases in all-cause mortality for both men and women aged 45-54. Disaggregating by cause, we find evidence automation is associated with increases in drug overdose deaths, suicide, homicide and cardiovascular mortality although patterns differ across age-sex groups. We go on to examine heterogeneity in effects by safety net program generosity, labor market policies, and the supply of prescription opioids.

\section{S-263 OMEGA-NET INVENTORY OF OCCUPATIONAL EXPOSURE ASSESSMENT TOOLS}

'Susan Peters, Danielle Vienneau, Alexia Sampri, Michelle Turner, Gemma Castaño Vinyals, Merete Bugge, Roel Vermeulen. 'Utrecht University, Netherlands

\subsection{6/OEM-2021-EPI.427}

Introduction The Network on the Coordination and Harmonisation of European Occupational Cohorts (OMEGA-NET) was set up to enable optimization of using industrial and general population cohorts across Europe to advance aetiological research. High quality harmonised exposure assessment is crucial for such international occupational health research.

Objective To facilitate an integrated research strategy, a concerted effort is needed to catalogue occupational exposure information. This study aims to provide a comprehensive overview of exposure assessment tools that could be used for occupational epidemiological studies.

Methods An online inventory was set-up to collect meta data on exposure assessment tools (https://occupationalexposuretools.net/). Occupational health researchers were invited via newsletters, editorials and individual mails to provide details on job-exposure matrices (JEMs), exposure databases, and occupational coding systems and crosswalks, with a focus on Europe.

Results Meta data on 38 JEMs and 9 national exposure databases had been collected up to May 2021. Most JEMs on which these data were entered were developed in the Netherlands and the Nordic countries. A wide variety of exposures was covered, with dusts and fibres (in 15 JEMs) being the most common types. Just a few JEMs covered biological factors (5) and employment conditions (1). Dusts and fibres were also the most common exposures in the databases (6 out of 9), followed by solvents and pesticides (both in 4 databases). Furthermore, information was collected on 24 occupational coding systems from more than 10 countries, indicating related systems as well as the availability of crosswalks or automated coding from free-text.

Conclusion This inventory forms the basis for a searchable web-based database of meta-data on existing occupational exposure information, so that researchers can find the available tools for assessing occupational exposures in their cohorts. This inventory remains open for further additions, to enlarge its coverage and include newly developed tools

\section{S-264 TAKING A WORKING LIFE EXPOSOME APPROACH: WHY DO WE NEED IT AND WHICH CHALLENGES ARE WE FACING?}

${ }^{1}$ Susan Peters, Anjoeka Pronk, Roel Vermeulen. 'Utrecht University, Netherlands

10.1136/OEM-2021-EPI.428

The exposome concept was conceived in 2005 as a way to represent non-genetic drivers of health and disease. The exposome encompasses all non-genetic risk factors experienced during a person's life (the external exposome) and its relation to biological responses inside the human body (the internal exposome). This concept was born out of the recognition that - while there was a revolution in our understanding on the genetic drivers of disease - our understanding on environmental drivers was much more limited. About 70$80 \%$ of the disease burden could probably be explained by the exposome.

Fifteen years after the exposome concept was introduced, several advances have been made in the quantification of the exposome by using combinations of different technologies (sensors, bioassays), large exposure and health datasets, and advanced statistical methods. These advances enable moving away from the 'one exposure-one disease' approach.

Although occupational risk factors are known to have an important impact on non-communicable diseases, the application of the exposome approach to occupational health has been limited. This is surprising as i) our working life covers a significant proportion of our total lifespan; ii) occupational exposures occur often in complex settings; iii) working-life encompasses important vulnerable life stages including the reproductive period; and iv) occupational exposures are closely related to lifestyle/behaviour (e.g. diet, physical activity, smoking and alcohol consumption) and socioeconomic status.

Given the varying exposures and interrelations with other factors across the life course a (holistic) network approach is needed to fully understand the impact of the working life exposome on health. The exposome approach and associated tools start to allow building a (partial) picture of the occupational exposome, which may lead to new insights on how the occupational exposome affects health and provide new leads for prevention. 